\title{
Hybrid Therapy for Metastatic Epidural Spinal Cord Compression: Technique for Separation Surgery and Spine Radiosurgery
}

\section{Ori Barzilai, MD* \\ Ilya Laufer, MD*§ \\ Adam Robin, MD* \\ Ran Xu, MD* ๆ \\ Yoshiya Yamada, MD \\ Mark H. Bilsky, MD* $§$}

*Department of Neurosurgery, Memorial Sloan-Kettering Cancer Center, New York, New York; ${ }^{\ddagger}$ Department of Radiation Oncology, Memorial Sloan-Kettering Cancer Center, New York, New York ${ }^{\S}$ Department of Neurological Surgery, Weill Cornell Medical College, New York

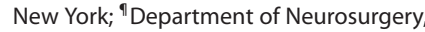
Charité - Universitätsmedizin Berlin, Berlin, Germany

\section{Correspondence:}

Mark H. Bilsky, MD,

Department of Neurosurgery,

Memorial Sloan Kettering Cancer Center, 1275 York Avenue,

New York, NY 10065.

E-mail: bilskym@mskcc.org

Received, July 24, 2017.

Accepted, June 4, 2018.

Published Online, June 8, 2018.

Copyright $(\odot) 2018$ by the

Congress of Neurological Surgeons
BACKGROUND: Despite major advances in radiation and systemic treatments, surgery remains a critical step in the multidisciplinary treatment of metastatic spinal cord tumors. OBJECTIVE: To describe the indications, rationale, and technique of "hybrid therapy" (separation surgery and concomitant spine stereotactic radiosurgery [SRS]) along with practical nuances.

METHODS: Separation surgery describes a posterolateral approach for circumferential epidural decompression and stabilization. The goal is to decompress the spinal cord, stabilize the spine, and create adequate separation between the neural elements and the tumor for SRS to achieve durable tumor control.

RESULTS: A transpedicular route to achieve ventrolateral access and limited resection of the tumorous vertebral body is carried out. In the setting of high-grade cord compression, caution must be taken when performing the tumor decompression. "Separation" of the ventral epidural tumor component anteriorly creates space for concomitant SRS while a simple laminectomy would not adequately achieve this goal. Dissection of the posterior longitudinal ligament allows maximal ventral decompression. Gross total tumor resection is not crucial for durable tumor control using the "hybrid therapy" model. Thus, attempts at ventral tumor resection may unnecessarily increase operative morbidity. Cement augmentation of the construct or vertebral body may improve construct stability. CT myelogram is the preferred exam for postoperative SRS planning. Radiosurgical planning constitutes a multidisciplinary effort and guidelines for contouring in the postoperative setting have recently become available.

CONCLUSION: Separation surgery is an effective, well-tolerated, and reproducible surgery. It provides safe margins for concomitant SRS. Combined, this "Hybrid Therapy" allows durable local control, maintenance of spinal stability, and palliation of symptoms, while minimizing operative morbidity.

KEY WORDS: Tumor, Separation Surgery, Radiosurgery, Hybrid therapy

Operative Neurosurgery 16:310-318, 2019

DOI: 10.1093/ons/opy137
A

pproximately $30-40$ percent of the oncologic population develops spinal metastases. ${ }^{1,2}$ At our center, a multi-

ABBREVIATIONS: BED, biologically effective tumor dose; CTV, clinical target volume; ESCC, epidural spinal cord compression; GTV, gross tumor volume; IOM, Intraoperative nerve monitoring; PTV, planning target volume; PMMA, poly-methylmethacrylate; PLL, posterior longitudinal ligament

Operative Neurosurgery Speaks! Audio abstracts available for this article at www.operativeneurosurgery-online.com.

Supplemental digital content is available for this article at www.operativeneurosurgery-online.com. disciplinary team of specialists evaluate spine tumor treatment using the previously published decision framework, NOMS. ${ }^{3,4}$ Surgery plays a critical role this treatment algorithm.

Recent data suggests that stereotactic body radiosurgery achieves local control of spine tumors while palliating associated symptoms. ${ }^{6-8}$ In a large single-center institutional experience from MSKCC, 811 spinal tumors treated with single-fraction stereotactic radiosurgery (SRS) were evaluated. This study demonstrated the significant local control advantage imparted by high-dose single-fraction SRS.? Importantly, local control rates at $2 \mathrm{yr}$ approached 98\%, 
TABLE 1. A Step by Step Guide to Separation Surgery

\begin{tabular}{|c|c|}
\hline Preparation & $\begin{array}{l}\text { Insertion of a Foley catheter, a-line, connection to INM. The patient is positioned prone and fluoroscopy used to localize the } \\
\text { skin incision. The surgical site is prepped and draped. }\end{array}$ \\
\hline Exposure & $\begin{array}{l}\text { Midline linear skin incision. Sub-periosteal muscle dissection exposing posterior spinal elements and the transverse processes } \\
\text { laterally. Verification of proper level. }\end{array}$ \\
\hline Decompression & Posterior elements are drilled with a high-speed 3-mm match-stick burr to expose ligamentum flavum and dura. \\
\hline Lateral exposure & The facet joints and bilateral pedicles are drilled until flush with vertebral body. \\
\hline Ventral separation & $\begin{array}{l}\text { Woodson dissector used to depress the epidural tumor component ventrally. When a large cavity is created in the vertebral } \\
\text { body, intraoperative cement augmentation can provide anterior column support. }\end{array}$ \\
\hline Confirmation & Ultrasound may be helpful in determining when adequate separation is achieved. \\
\hline Closure & Meticulous hemostasis achieved, subfascial drain placed and the wound is sutured layer by layer. \\
\hline
\end{tabular}

independent of tumor histology. Maximizing treatment dose and avoiding radiation-induced spinal cord injury requires a safe distance between the radiation target and the spinal cord; thus, patients with high-grade epidural spinal cord compression $(\text { ESCC })^{10}$ are not candidates for "up-front" radiation treatment. ${ }^{11}$ The goal of separation surgery is to create distance between the tumor and spinal cord creating a safe target for the delivery SRS with maximization of the biologically effective tumor dose (BED). ${ }^{3}$ Herein the authors describe the "hybrid therapy" approach with detailed surgical and radiosurgical nuances and case examples. To note, as this is a technical manuscript and no patient data are presented, IRB/ethical committee approval or informed consent were not required. This manuscript is a "stepby-step" guide (Table 1) to assist others in treating patients with metastatic ESCC.

\section{OPERATIVE TECHNIQUE FOR SEPARATION SURGERY}

Patients are placed under general anesthesia and an arterial line and Foley catheter are placed. Intraoperative nerve monitoring (INM) is routinely used including EMGs, SSEPs, and MEPs.

Following fluoroscopic localization, a midline linear skin incision is performed and the posterior spinal elements are exposed using monopolar cautery. Posterior pedicle screw-rod constructs are placed prior to the decompression to avoid manipulation of spinal hardware over the exposed spinal cord. All patients who undergo separation surgery will need spinal instrumentation and fixation since the vertebral body integrity is usually compromised by tumor invasion and decompression requires removal of the lamina and pedicle/joint complex. The potential arthrodesis is severely compromised in oncologic patients due to poor bone quality, radiation and chemotherapy. ${ }^{12}$ The need for multiple levels of decompression and adjacent level involvement are not uncommon. Hence, it is our common practice to insert long segment fixation, typically 2 levels above and 2 levels below the tumor while skipping the index level (Table 1). Pedicle screws can be placed using various navigation systems ${ }^{13}$ or by standard "freehand" insertion. Rods are contoured and tightened to complete the instrumentation process. Shorter constructs using percutaneous muscle-sparing technique and screw-cement augmentation are optional.

Various techniques have been described for performing posterolateral decompression of the spinal canal. Due to highgrade ESCC, it is crucial to avoid transmitting pressure to the spinal cord during decompression. The posterior elements are drilled with a high-speed 3-mm matchstick bur. The bony structures are thinned and the ligamentum flavum is resected. A surgical corridor to the ventral epidural space is created via bilateral drilling of the pedicles and facet joints as needed (Figure 1). The dissection is initiated in a relatively normal anatomical plane and extended towards the site of maximal compression. Tumor is resected circumferentially using tenotomy scissors (Figure 2). To avoid damage to the spinal cord, the ventral component of the tumor is dissected away from the dura. This is achieved after a partial vertebrectomy is performed. Typically removing approximately $20 \%$ of the involved vertebral body is sufficient. Once a ventral cavity has been created, a "Woodson" dissector is used to depress the epidural component ventrally and free the anterior part of the spinal canal (Figure 3). If a large portion of the vertebral body is removed, anterior support can be achieved by inserting poly-methyl-methacrylate (PMMA) into the anterior vertebral cavity.

One of the greatest challenges of separation surgery is achievement of adequate ventral decompression. The anterior dura is connected to the posterior longitudinal ligament (PLL) via the epidural ligaments of Hoffman. ${ }^{14}$ Hence, resection of the PLL and separating these anterior attachments is routinely carried out (Figure 4). Ensuring optimal ventral 


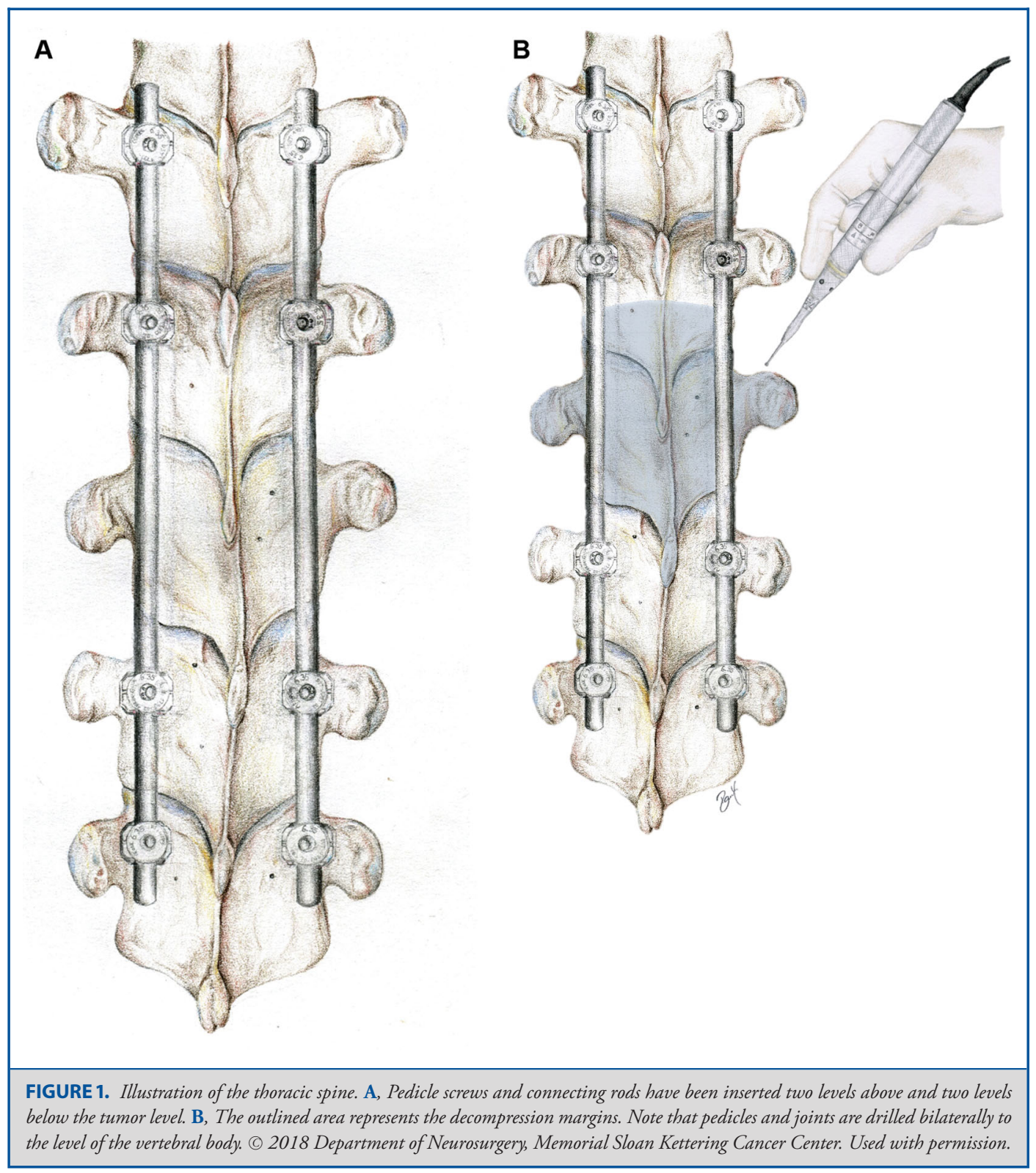

separation can be achieved intraoperatively using an ultrasound (Figure 5).

Importantly, aggressive or gross-total resection of the vertebral body or paraspinal tumor is not required since postoperative SRS will effectively treat these tumor components. Hemostasis is achieved and the wound is irrigated copiously. To optimize arthrodesis, the facet joints and transverse processes are decorticated and local bone graft is placed. Often, when the vertebral body is compressed, kyphoplasty/vertebroplasty to the treatment level is performed for pain palliation. ${ }^{15}$ Additionally, the injection of bone cement through fenestrated pedicle screws can aid in bony purchase and minimize hardware failure. ${ }^{16,17}$ Finally, a drain is placed in the epidural space and the surgical site is sutured in multiple layers (Figure 6).

\section{SRS Treatment}

Spine radiosurgery is the second part of the "hybrid therapy" concept. It is important to acknowledge the symbiotic relationship between surgical decompression and SRS. This synergy comes from utilizing these 2 modalities as equal parts of one treatment plan for high-grade ESCC from metastatic spine disease. Without surgical decompression, adequate tumoricidal SRS doses cannot be delivered to the entire tumor volume within the constraints of spinal cord tolerance. Planning for SRS typically 


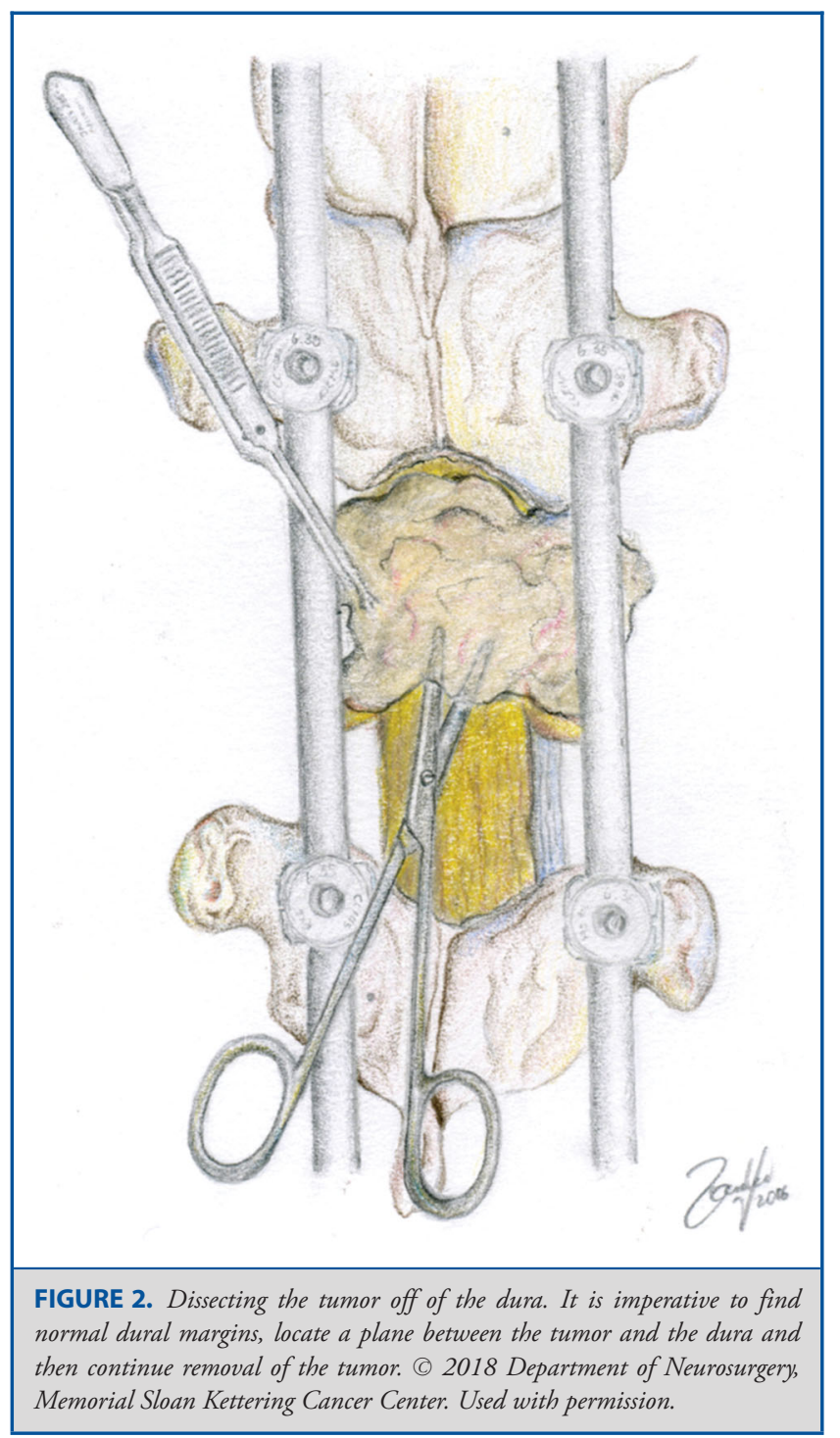

begins while the patient is still in the hospital and recovering from surgery.

\section{Simulation}

Following surgery, MRI-related artifact from hardware can limit optimal radiosurgical treatment planning. Therefore, all patients undergo CT-myelography that allows better visualization of the neural elements, construct and organs at risk (OARs), ie, spinal cord. Better visualization translates into better contouring of conformal radiation allowing safe delivery of maximal treatment dose. It is often beneficial to keep patients on intravenous patient-controlled analgesia in the postoperative setting until after the myelogram is completed. Patients are immobilized during simulation in a reproducible manner and a nonerasable ink mark is made on the patient's skin to ensure consistent repositioning for treatment. Immobilization of the

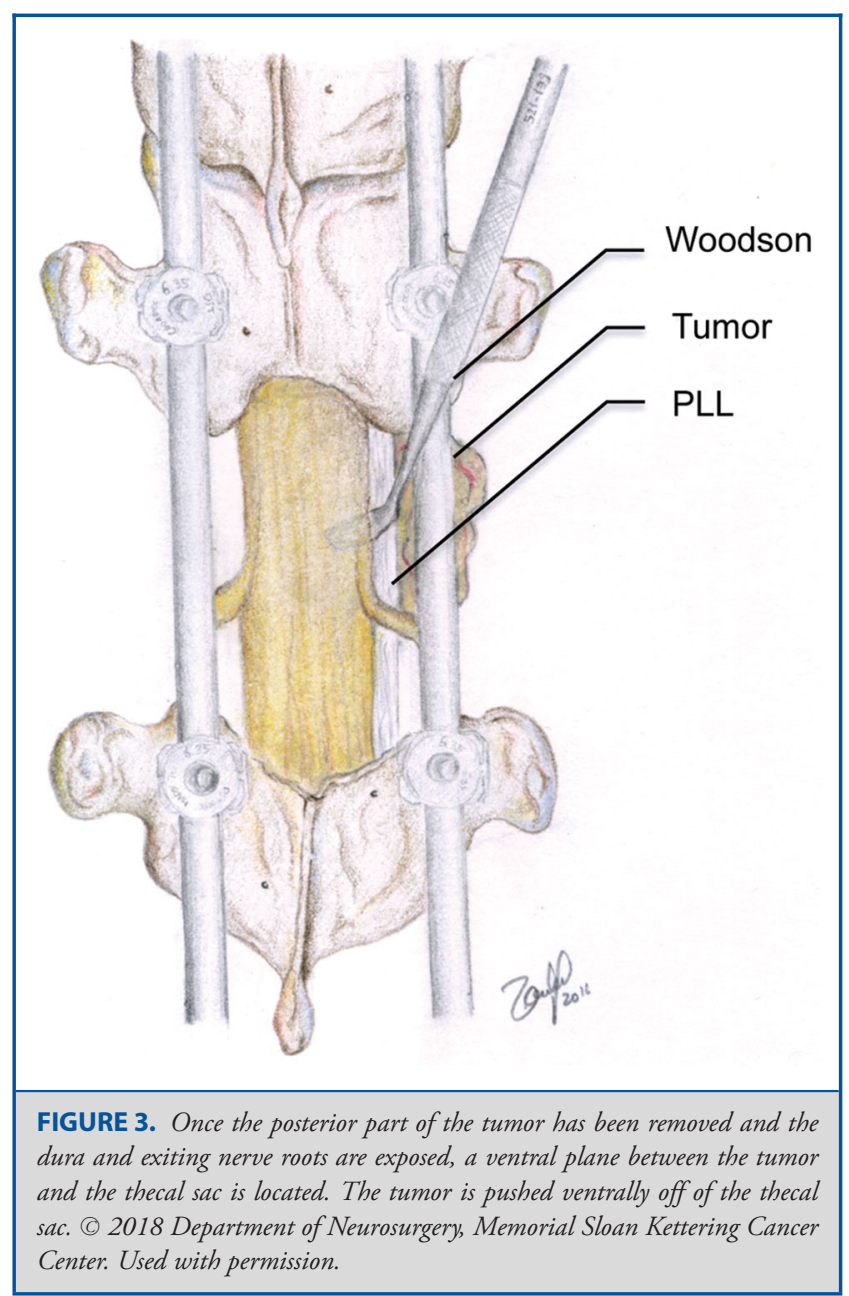

patient can be done with the use of rigid external immobilization devices, by active motion detection and compensation during treatment delivery and by fast treatment delivery. ${ }^{18-20}$ Preoperative images are fused to postoperative simulation imaging for accurate delineation of tumor and OARs. Visualization of the tumor and delineation of OARs can be facilitated by contrast enhanced imaging. In many centers, when contrast injection is planned, an additional noncontrast CT is performed for treatment planning to eliminate uncertainty in dose computation caused by the contrast. ${ }^{21}$ We normally obtain a preoperative MRI with contrast enhancement to visualize the tumor, yet also rely heavily on noncontrast T1 images for tumor delineation and MR STIR images for evaluation of mechanical integrity. These images are subsequently fused to the postoperative, thin cut, CT myelography imaging for radiation planning purposes.

\section{Contouring}

In 2012, the Spine Radiosurgery Consensus Consortium released contouring guidelines for spinal SRS. ${ }^{22}$ Target volumes are defined according to the definitions set by the International 

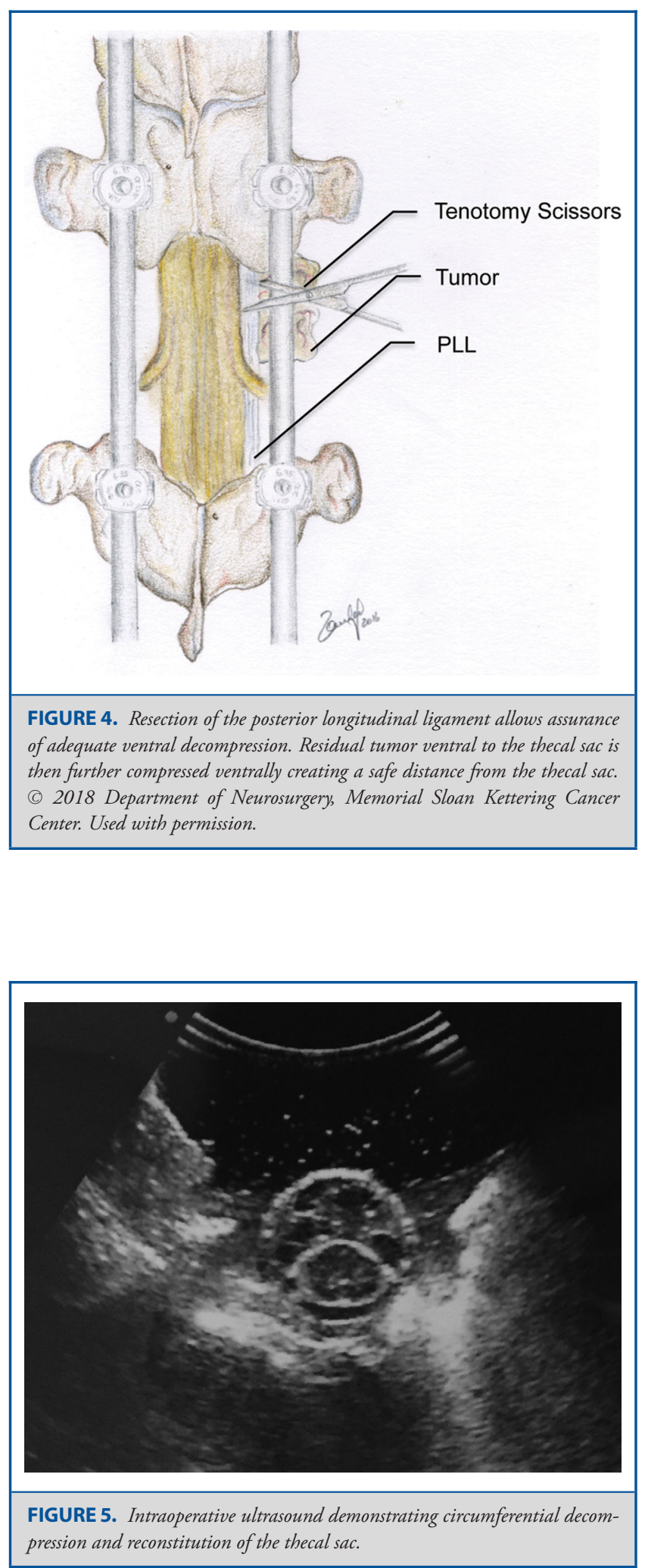

Commission on Radiation Units and Measurements Report 50.23 The gross tumor volume (GTV) represents palpable disease at surgery or gross tumor seen on imaging. The clinical target volume (CTV) is the region of potential microscopic spread of tumor cells that includes the GTV. Hence the CTV represents the volume that needs to be sterilized of tumor cells. The planning target volume (PTV) is a geometric construct that encompasses the CTV and adds an additional margin of tissue to ensure that the CTV receives the intended dose. This margin is necessary to take into account factors that are difficult to control exactly such as the uncertainty of patient positioning and motion during treatment, organ motion during treatment, physical errors of the treatment machinery and other random errors that can occur. In modern stereotactic spine radiosurgery, a typical PTV expansion on the CTV is $2 \mathrm{~mm}$.

More recently, guidelines became available for contouring and treatment planning in the postoperative setting ${ }^{24,25}$ (Figure 7). Due to the complexity of decision making involved in these cases, at our institution treatment plans are always formulated in a multidisciplinary fashion comprised of radiation oncology and neurosurgery teams.

\section{Treatment Planning}

Treatment planning is performed based upon the prescribed dose and the allowable dose to surrounding normal structures. In general, treatment planning for spine radiosurgery is similar to principles employed in cranial radiosurgery. For example, dose uniformity within the target volume is sacrificed for steep dose gradients immediately outside the target volume to allow maximal sparing of nearby dose sensitive structures such as the spinal cord or esophagus, and radiation "hot spots" over $130 \%$ of the prescribed dose are allowed. An ideal treatment plan would be able to cover at least $90 \%$ of the PTV with the prescribed dose, but better than $80 \%$ coverage of the PTV with the prescribed dose would be still considered acceptable.

\section{DISCUSSION}

"Hybrid Therapy" is a combined treatment paradigm utilizing separation surgery combined with early postoperative SRS for MESCC. This therapeutic model has been previously established as a safe and effective treatment. ${ }^{26}$

The putative role of surgery was established in 2005 with the landmark study by Patchell et $\mathrm{al},{ }^{5}$ which demonstrated that decompressive spinal surgery combined with radiation offered a survival benefit, improved ambulation, less dependence on opioids and better local control when compared to radiation alone. A recent review described several common posterior approaches for tumor decompression of the spinal canal. ${ }^{27}$ In the review, the authors analyze a variety of posterior surgeries for spinal metastases, including laminectomy with or without instrumentation for stabilization, transpedicular corpectomy, and costotransversectomy. Considering the gamut of surgical 

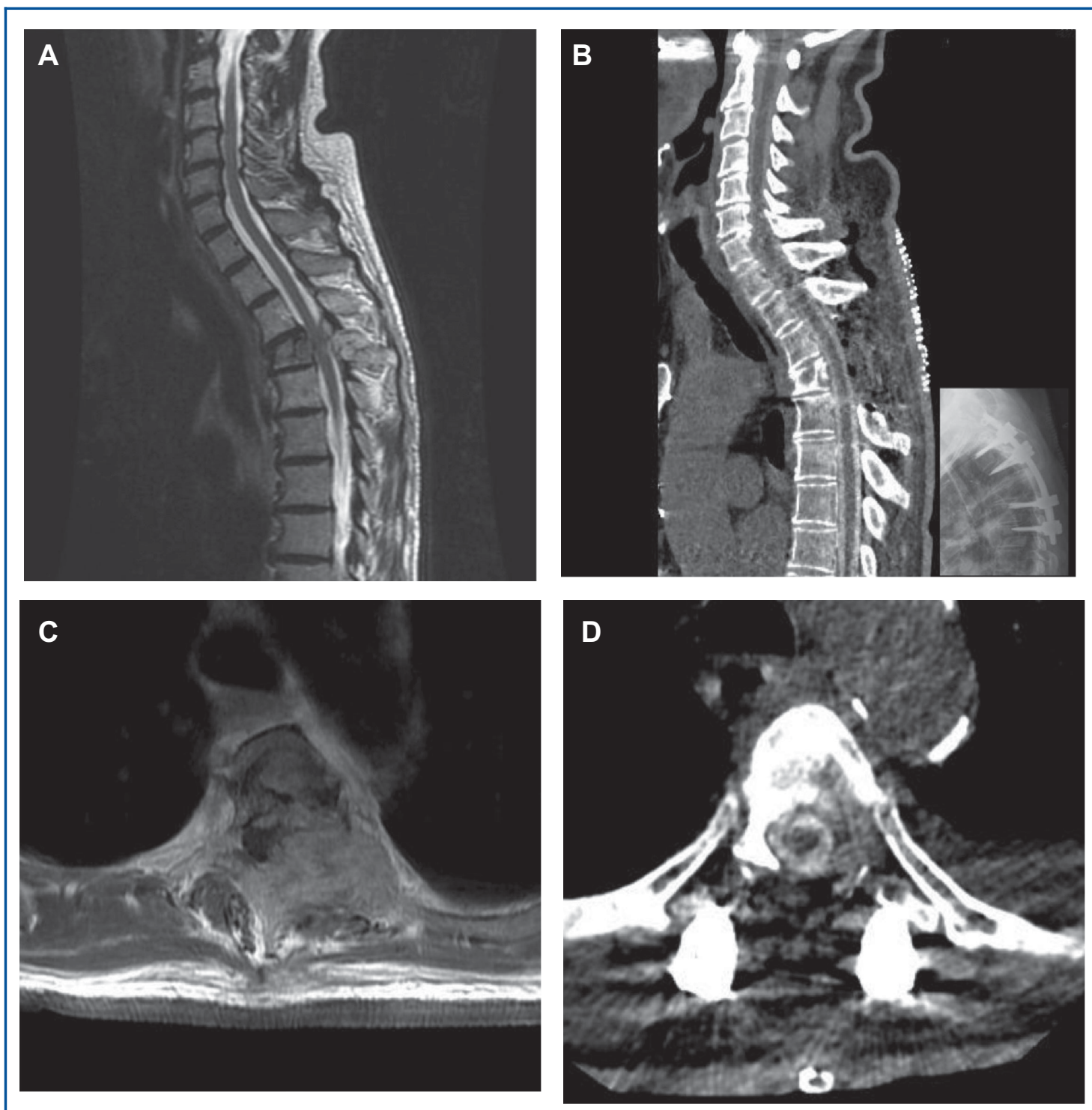

FIGURE 6. Representative case example. A 76-yr-old male with renal cell carcinoma presented with severe back pain and no signs of myelopathy. Evaluation including spinal imaging demonstrated a T4 tumor with high grade cord compression. A, and B, Pre and postoperative sagittal imaging. A, Preoperative MRI T2 demonstrating a T4 tumor. B, Postoperative CT myelography demonstrating the surgical and reconstituted thecal sac. $\mathbf{C}$ and $\mathbf{D}$, Pre and postoperative axial imaging. C, Preoperative MRI T1 with contrast enhancement. D, Postoperative CT myelography. Note the high-grade cord compression preoperatively and the cerebrospinal fluid-filled thecal sac postoperatively. This new distance between the tumor and the spinal cord allows for safe concurrent radiosurgical treatment.

procedures ranges from least to most aggressive, separation surgery lies in the middle of the spectrum.

Separation surgery was first described in 2000 as a singlestage posterolateral transpedicle approach for spondylectomy, epidural decompression, and circumferential fusion for treatment of spinal metastases. ${ }^{28}$ Since significant knowledge has been garnered with extensive data evaluating the safety, efficacy and adverse effects of this surgery. ${ }^{3,26,29}$ In our previous analysis of 186 patients undergoing hybrid therapy, patients who were post operatively treated with single fraction $24 \mathrm{~Gy}$ and high-dose hypofractionated ( 3 fractions of $8 \mathrm{~Gy}$ ) had 1 yr recurrence rates of $9 \%$ and $4.1 \%$, respectively. ${ }^{4}$ Notably, nearly $80 \%$ had radioresistant tumor histologies and about half had failed prior radiotherapy. In that cohort, no patient suffered a neurological complication. In another retrospective analysis of 318 patients who underwent posterolateral decompression and posterior screw-rod 

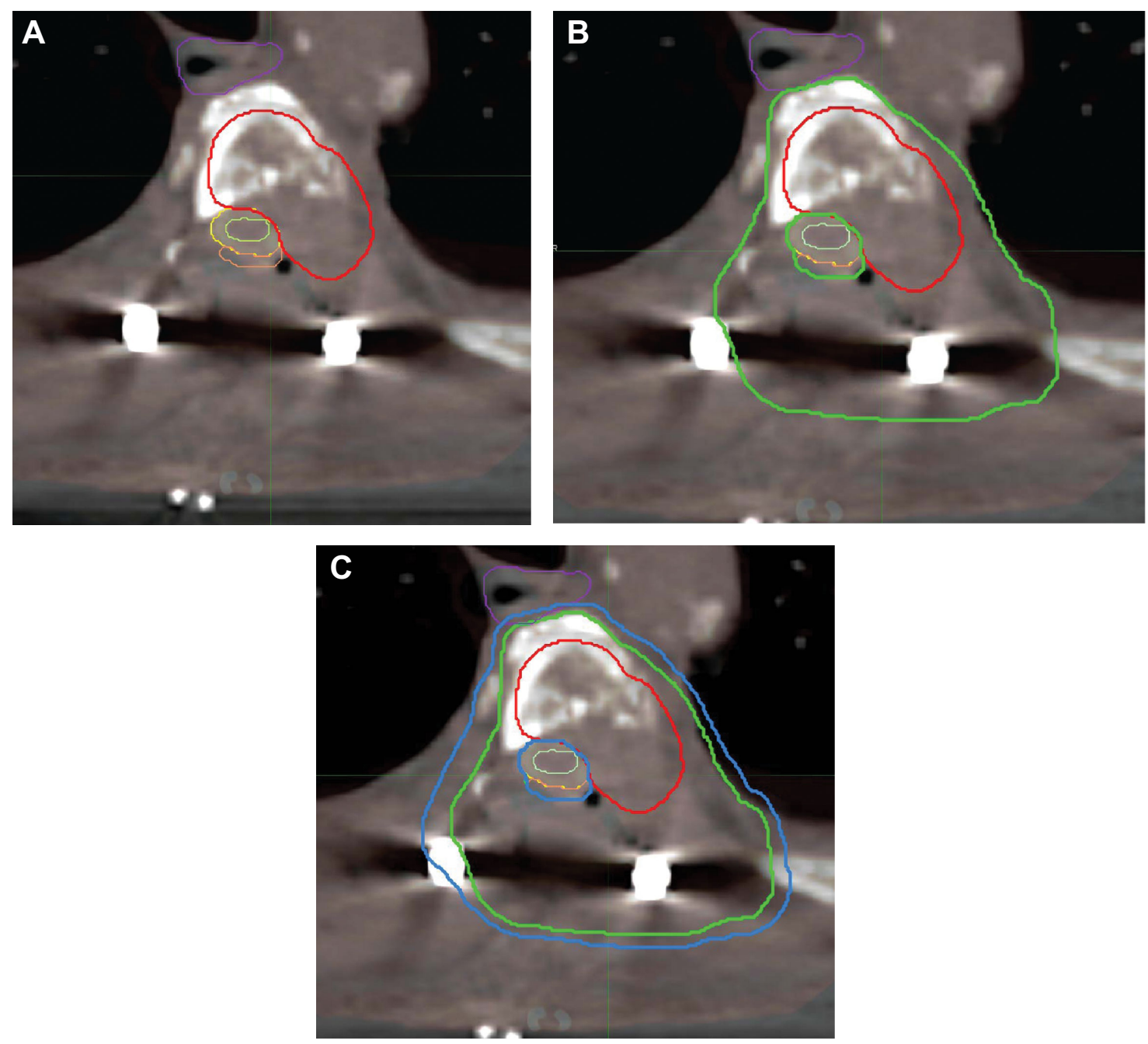

FIGURE 7. Postoperative CT myelography at T4 demonstrating contouring for the patient described in Figure 6. Contouring of the GTV in the postoperative setting is done using preoperative imaging and the CTV is delineated accordingly. OARs are outlined fusing preoperative to postoperative CT myelogram or MR scans. The CTV includes abnormal marrow signal suspicious for microscopic invasion and an adjacent normal bony expansion to account for subclinical tumor spread in the marrow space. To note, in the case presented, a small space posterior to the dura (orange) is outlined as a P32 brachytherapy plaque was placed on the dura during surgery delivering radiation to the posterior dural margin intraoperatively and allowing for optimal dose delivery postoperatively. ${ }^{36,37} \mathbf{A}$, The GTV is outlined in red. $\mathbf{B}$, The G=CTV is outlined in green. C, The PTV is outlined in blue. The OARs are outlined as follows; esophagus in purple, spinal cord in light green, dura in yellow. $O A R s=$ organs at risk, GTV = gross tumor volume, $C T V=$ clinical target volume, PTV = planning target volume.

fixation without supplemental anterior fixation, $2.8 \%$ exhibited signs and symptoms of hardware failure and required revision of the instrumentation. ${ }^{29}$

Advancements in radiation technology allow delivery of highdose conformal radiation to precise areas while minimizing exposure to vital organs such as the spinal cord. Spinal SRS is a valid therapeutic option for durable palliation and local progression-free survival..$^{30,31}$ However, when the epidural tumor component is significant, delivering high doses of radiation without compromising the spinal cord is not feasbile. ${ }^{10}$ Hence, even with advancements in adjuvant cancer treatments, surgery retains a critical role. As mentioned above, guidelines are available for radiosurgery planning and contouring in both the up-front as well as the postoperative setting. It is important to acknowledge that some controversy remains regarding the optimal delivered dose. Further, spinal cord toxicity remains a problem, particularly in the setting of circumferential tumor infiltration and previously irradiated targets..$^{1,32-34}$ One solution is to deliver a therapeutic 
dose to the dural margin is the use of single-dose intraoperative brachytherapy. ${ }^{35}$ The P32 plaque delivers a high dose with a steep dose-fall off making it an ideal dural radiation plaque. In small series, P32 has been shown to a useful adjunct to surgical intervention following epidural decompression. ${ }^{36-38}$

Every case must be considered on an individual basis, and following a decision-making framework is helpful. ${ }^{3}$ The authors use the NOMS framework which is an adaptable framework that considers the neurological, oncological, mechanical stability, and systemic status of a patient and allows for the adaptation of new and evolving treatments into the model. ${ }^{4}$ Surgical indications that are heavily weighed are the presence of a neurological deficit, ESCC, ${ }^{10}$ and spinal instability. ${ }^{39}$ The goals of treatment, overall tumor burden, functional status and co-morbidity burden are considered before recommending surgical treatment.

There are a several important technical nuances to consider when performing separation surgery. Although this is a posterior only approach, a simple laminectomy for decompression is typically inadequate as it will not provide circumferential decompression. Without adequate ventral decompression, safe delivery of tumoricidal-radiation doses will not be possible. We have found that resection of the PLL and bilateral ventral separation, as described herein, is necessary for optimal postoperative SRS. Intraoperative confirmation of ventral separation can be easily achieved with ultrasonography as shown in Figure 5.

Some topics that remain debatable: (1) Electing to perform separation surgery does not eliminate the role for anterior approaches. In the authors' experience, we have found that it is possible to achieve all treatment goals rapidly and efficiently through this posterolateral approach. The goal remains the same- - rapid and safe circumferential spinal cord decompression laying the ground for radiosurgery. (2) Providing anterior column support when necessary can be achieved safely by reconstructing the anterior column using PMMA. ${ }^{40}$ (3) To our knowledge, there are no data supporting deformity corrections secondary to pathologic fractures in the cancer population. As such, the authors believe that for cancer patients, the benefit of minimizing the extent of surgery outweighs the risks. Future studies will determine the risk-benefit ratio from these larger-scale corrective operations. (4) It is possible that with new cancer therapies allowing extended overall survival, patients will experience more construct failures or other long-term complications. Strategies to provide more durable constructs such as cement augmented screws are currently being evaluated, but the efficacy of these strategies has not been fully determined. (5) The optimal timing of surgery is unclear. Treatment of MESCC requires action to prevent development or progression of neurologic deficits and facilitate return to systemic therapy. The duration and severity of neurologic deficit predict neurological recovery in patients with MESCC. ${ }^{41}$ Hence, efforts to reduce the duration of ambulation loss and to prevent progression of neurologic deficits should be made. (6) Bone quality in cancer patients is typically poor due to the osteolytic metastases, chemotherapy, radiation, and other co-morbidities such as osteoporosis. Variable fusion rates in this population are reported (36\%-100\%) and various options for bone graft are used according to surgeon's preference. $^{42}$ (7) Lastly, the authors routinely use intraoperative electrophysiological monitoring, which offers valuable intraoperative knowledge of the integrity of the motor and sensory tracts. ${ }^{43}$ There is no level 1 evidence to support the routine use of INM and in case of urgent need of spinal cord decompression; the absence of INM should not delay surgery.

\section{CONCLUSION}

Despite tremendous advancements in the care of the patient with a metastatic spine tumor, particularly SRS, surgery continues to play a key role in the treatment paradigm. Although multiple surgical approaches exist, separation surgery is a posterolateral approach that provides rapid decompression, stabilization, and allows for rapid continuation of treatment. Assuring adequate circumferential epidural decompression is crucial, and allows for optimal SRS planning. Hybrid therapy is an effective, tolerable, and reproducible treatment.

\section{Disclosures}

This research was funded in part through the NIH/NCI Cancer Center Support Grant P30 CA008748. Dr Laufer is a consultant for Depuy/Synthes, Globus, and SpineWave. Dr Yamada is a consultant for Varian Medical Systems and a Medical Advisory Board Member for the Chordoma Foundation. Dr Bilsky is a consultant for Globus and Brainlab and receives royalties from Depuy/Synthes. The authors have no personal, financial, or institutional interest in any of the drugs, materials, or devices described in this article.

\section{REFERENCES}

1. Klimo P Jr, Kestle JR, Schmidt MH. Clinical trials and evidence-based medicine for metastatic spine disease. Neurosurg Clin N Am. 2004;15(4):549-564.

2. Ortiz Gomez JA. The incidence of vertebral body metastases. Int Orthop. 1995;19(5):309-311.

3. Bilsky M, Smith M. Surgical approach to epidural spinal cord compression. Hematol Oncol Clin North Am. 2006;20(6):1307-1317.

4. Laufer I, Rubin DG, Lis E, et al. The NOMS framework: approach to the treatment of spinal metastatic tumors. Oncologist. 2013;18(6):744-751.

5. Patchell RA, Tibbs PA, Regine WF, et al. Direct decompressive surgical resection in the treatment of spinal cord compression caused by metastatic cancer: A randomised trial. Lancet North Am Ed. 2005;366(9486):643-648.

6. Guckenberger M, Mantel F, Gerszten PC, et al. Safety and efficacy of stereotactic body radiotherapy as primary treatment for vertebral metastases: a multiinstitutional analysis. Radiat Oncol. 2014;9(1):226.

7. Bhattacharya IS, Hoskin PJ. Stereotactic body radiotherapy for spinal and bone metastases. Clin Oncol. 2015;27(5):298-306

8. Hanna GG, Landau D. Stereotactic body radiotherapy for oligometastatic disease. Clin Oncol. 2015;27(5):290-297.

9. Yamada Y, Katsoulakis E, Laufer I, et al. The impact of histology and delivered dose on local control of spinal metastases treated with stereotactic radiosurgery. Neurosurg Focus. 2017;42(1):E6.

10. Bilsky MH, Laufer I, Fourney DR, et al. Reliability analysis of the epidural spinal cord compression scale. J Neurosurg Spine. 2010;13(3):324-328.

11. Lovelock DM, Zhang Z, Jackson A, et al. Correlation of local failure with measures of dose insufficiency in the high-dose single-fraction treatment of bony metastases. Int J Radiat Oncol Biol Phys. 2010;77(4):1282-1287.

12. Rades D, Fehlauer F, Schulte R, et al. Prognostic factors for local control and survival after radiotherapy of metastatic spinal cord compression. J Clin Oncol.. 2006;24(21):3388-3393. 
13. Costa F, Dorelli G, Ortolina A, et al. Computed tomography-based image-guided system in spinal surgery: state of the art through 10 years of experience. Neurosurgery. 2015;11:(Suppl 2):59-67.

14. Tardieu GG, Fisahn C, Loukas M, et al. The epidural ligaments (of hofmann): A comprehensive review of the literature. Cureus. 2016;8(9):e779.

15. Fourney DR, Schomer DF, Nader R, et al. Percutaneous vertebroplasty and kyphoplasty for painful vertebral body fractures in cancer patients. I Neurosurg. 2003;98(1 Suppl):21-30.

16. Moussazadeh N, Rubin DG, McLaughlin L, Lis E, Bilsky MH, Laufer I. Short-segment percutaneous pedicle screw fixation with cement augmentation for tumor-induced spinal instability. Spine J. 2015;15(7):1609-1617.

17. Frankel BM, Jones T, Wang C. Segmental polymethylmethacrylate-augmented pedicle screw fixation in patients with bone softening caused by osteoporosis and metastatic tumor involvement: a clinical evaluation. Neurosurgery. 2007;61(3):531538.

18. Lo SS, Fakiris AJ, Chang EL, et al. Stereotactic body radiation therapy: A novel treatment modality. Nat Rev Clin Oncol 2010;7(1):44-54.

19. Foote M, Bailey M, Smith L, et al. Guidelines for safe practice of stereotactic body (ablative) radiation therapy. J Med Imaging Radiat Oncol. 2015;59(5):646-653.

20. Li W, Sahgal A, Foote M, Millar BA, Jaffray DA, Letourneau D. Impact of immobilization on intrafraction motion for spine stereotactic body radiotherapy using cone beam computed tomography. Int J Radiat Oncol Biol Phys. 2012;84(2):520-526.

21. Lo SS, Foote M, Siva S, et al. Technical know-how in stereotactic ablative radiotherapy (SABR). J Med Radiat Sci. 2016;63(1):5-8.

22. Cox BW, Spratt DE, Lovelock $M$, et al. International spine radiosurgery consortium consensus guidelines for target volume definition in spinal stereotactic radiosurgery. Int J Radiat Oncol Biol Phys. 2012;83(5):e597-e605.

23. International Commission on Radiation Units and Measurements (ICRU) Report 50. https://icru.org/home/reports/prescribing-recording-and-reporting-photonbeam-therapy-report- 50 .

24. Redmond KJ, Robertson S, Lo SS, et al. Consensus contouring guidelines for postoperative stereotactic body radiation therapy for metastatic solid tumor malignancies to the spine. Int J Radiat Oncol Biol Phys. 2017;97(1):64-74.

25. Redmond KJ, Lo SS, Soltys SG, et al. Consensus guidelines for postoperative stereotactic body radiation therapy for spinal metastases: results of an international survey. J Neurosurg Spine. 2017;26(3):299-306.

26. Laufer I, Iorgulescu JB, Chapman T, et al. Local disease control for spinal metastases following "separation surgery" and adjuvant hypofractionated or highdose single-fraction stereotactic radiosurgery: outcome analysis in 186 patients. J Neurosurg Spine. 2013;18(3):207-214.

27. Molina C, Rory Goodwin C, Abu-Bonsrah N, Elder BD, De la Garza Ramos R, Sciubba DM. Posterior approaches for symptomatic metastatic spinal cord compression. Neurosurg Focus. 2016;41(2):E11.

28. Bilsky MH, Boland P, Lis E, Raizer JJ, Healey JH. Single-stage posterolateral transpedicle approach for spondylectomy, epidural decompression, and circumferential fusion of spinal metastases. Spine. 2000;25(17):2240-2250.

29. Amankulor NM, Xu R, Iorgulescu JB, et al. The incidence and patterns of hardware failure after separation surgery in patients with spinal metastatic tumors. Spine J. 2014;14(9):1850-1859.

30. De Bari B, Alongi F, Mortellaro G, Mazzola R, Schiappacasse L, Guckenberger M. Spinal metastases: Is stereotactic body radiation therapy supported by evidences? Crit Rev Oncol Hematol. 2016;98:147-158.

31. Tao R, Bishop AJ, Brownlee Z, et al. Stereotactic Body Radiation Therapy for Spinal Metastases in the Postoperative Setting: A Secondary Analysis of Mature Phase 1-2 Trials. Int J Radiat Oncol Biol Phys. 2016;95(5):1405-1413.

32. Yamada Y, Bilsky MH, Lovelock DM, et al. High-dose, single-fraction imageguided intensity-modulated radiotherapy for metastatic spinal lesions. Int J Radiat Oncol Biol Phys. 2008;71(2):484-490.

33. Ryu S, Jin JY, Jin R, et al. Partial volume tolerance of the spinal cord and complications of single-dose radiosurgery. Cancer. 2007;109(3):628-636.
34. Emami B, Lyman J, Brown A, et al. Tolerance of normal tissue to therapeutic irradiation. Int J Radiat Oncol Biol Phys. 1991;21(1):109-122.

35. Willett CG, Czito BG, Tyler DS. Intraoperative radiation therapy. JCO. 2007;25(8):971-977

36. Folkert MR, Bilsky MH, Cohen GN, et al. Intraoperative 32P high-dose rate brachytherapy of the dura for recurrent primary and metastatic intracranial and spinal tumors. Neurosurgery. 2012;71(5):1003-1011.

37. Folkert MR, Bilsky MH, Cohen GN, et al. Local recurrence outcomes using the $32 \mathrm{P}$ intraoperative brachytherapy plaque in the management of malignant lesions of the spine involving the dura. Brachytherapy. 2015;14(2):202-208.

38. Liu JK, Laufer I, Bilsky MH. Update on management of vertebral column tumors. CNS Oncology. 2014;3(2):137-147.

39. Fisher CG, DiPaola CP, Ryken TC, et al. A Novel classification system for spinal instability in neoplastic disease. Spine. 2010;35(22):E1221-E1229.

40. Zuckerman SL, Rao G, Rhines LD, McCutcheon IE, Everson RG, Tatsui CE. Interbody distraction and vertebral body reconstruction with polymethylmethacrylate for the treatment of pathological fractures. I Neurosurg Spine. 2017;27(6):700-708.

41. Laufer I, Zuckerman SL, Bird JE, et al. Predicting neurologic recovery after surgery in patients with deficits secondary to MESCC: Systematic review. Spine. 2016;41:(Suppl 20):S224-S230.

42. Elder BD, Ishida W, Goodwin CR, et al. Bone graft options for spinal fusion following resection of spinal column tumors: systematic review and meta-analysis. Neurosurgical Focus. 2017;42(1):E16.

43. Avila EK, Elder JB, Singh P, Chen X, Bilsky MH. Intraoperative neurophysiologic monitoring and neurologic outcomes in patients with epidural spine tumors. Clin Neurol Neurosurg. 2013;115(10):2147-2152.

Operative Neurosurgery Speaks! Audio abstracts available for this article at www.operativeneurosurgery-online.com.

Supplemental digital content is available for this article at www.operativeneurosurgery-online.com.

Supplemental Digital Content. Video. A step by step guide to separation surgery.

\section{COMMENT}

$\mathrm{n}$ this article, the authors eloquently describe their technique for separation surgery followed by stereotactic radiosurgery for spinal metastases. With the use of illustrations and video, the technique is clearly demonstrated for surgeons not familiar with these concepts. The authors emphasize the need for circumferential decompression of the spinal cord, which must include resecting the PLL and "separating" epidural tumor and retropulsed bone away from the ventral surface of the dura and spinal cord. Leaving compression ventral to the dura is not sufficient from a decompression standpoint and further limits the effectiveness of planned stereotactic radiosurgery afterwards. The key principle here is to re-establish a margin of tumor-free space around the thecal sac to maximize conformal radiation dose delivery. The authors are commended for their work and contribution.

John H. Shin

Boston, Massachusetts 